\title{
A CPSS-based reflectarray cell with reconfigurable capabilities
}

\author{
S. Mener ${ }^{1}$, R. Gillard ${ }^{1}$, R. Sauleau ${ }^{2}$, C. Cheymol $^{3}$, P. Potier $^{4}$ \\ ${ }^{1}$ : European University of Brittany, INSA, IETR, UMR CNRS 6164, 35708 Rennes, simon.mener@insa-rennes.fr, \\ raphael.gillard@insa-rennes.fr \\ 2 : University of Rennes 1, IETR, UMR CNRS 6164, 35042 Rennes, ronan.sauleau @univ-rennes1.fr \\ ${ }^{3}$ : French space agency CNES, 31401, Toulouse CEDEX 9, France, Cecile.Cheymol@cnes.fr \\ ${ }^{4}$ : DGA Maitrise de l'information, BP 57419, 35174, Bruz CEDEX, France, patrick.potier@dga.defense.gouv.fr
}

\begin{abstract}
A reconfigurable circular polarisation selective surface (CPSS) is proposed as a first step towards the design of dualcircular polarisation reflectarray with active and independent control in both polarisations. A CPSS unit-cell is studied at 8.5 GHz. It provides a 2-bit phase resolution in reflection for the left hand circular polarisation (LHCP) and is nearly transparent in right hand circular polarisation (RHCP). The simulated characteristics are excellent over a $6.4 \%$ bandwidth.
\end{abstract}

Keywords- Circular polarisation selective surface; Unit-cell; Reflectarray.

\section{INTRODUCTION}

Reconfigurable reflectarrays are very attractive for beam scanning or beam-shaping in space applications such as Earth observations and satellite communications. In such cases, circular polarisation $(\mathrm{CP})$ is usually preferred to prevent from losses due to polarisation rotation. Several circularly-polarised unit-cells have been proposed in the literature using either a linearly-polarised [1] or a circularly-polarised [2] incident wave. However, reconfigurable unit-cells with dual circular polarisations are still required in order to increase the datarates.

In this paper, we propose to use a circular polarisation selective surface (CPSS)-based unit-cell to provide a dualcircular polarisation operation. Fig. 1 illustrates the operation principle of the proposed geometry. The reflectarray consists of a two-layer structure. The upper one is a CPSS reflecting LHCP with a controllable reflection phase. RHCP is transmitted to a second buried layer that reflects it with an independent controllable phase-shift. This second layer can rely on standard CP unit-cells [3] as it only deals with a single polarisation. Here, we focus our attention on the first layer only, with the objective to achieve simultaneously i) a perfect separation of the two impinging circular polarisations, and ii) a 2-bit phase-shifting of the reflected LHCP polarisation.

\section{DESCRIPTION OF THE UNIT-CELL}

The basic Left-Handed CPSS (LH-CPSS) cell is depicted in Fig. 2. As mentioned in [4], an ideal LH-CPSS is a reciprocal, lossless, perfectly selective, symmetrical structure. The chosen LH-CPSS consists of a $1 \lambda$-long resonant wire, folded into 3 segments. The transverse segments (along $x$ and $y$-axes respectively) are $3 \lambda / 8$ long and are connected by a $\lambda / 4$ long longitudinal segment (along $z$-axis). This quarter wavelength separation ensures that the currents induced on the transverse segments by an impinging wave under normal incidence are in phase or out of phase, depending on the hand of the incident $\mathrm{CP}$ wave. One of the four possible phase-states of the proposed reconfigurable unit-cell is represented in Fig. 3 (the three remaining configurations will be explained in Figs. 4 to 6$)$. The transverse segments are printed $(18 \mu \mathrm{m}-$ thick) on two thin layers $(0.127 \mathrm{~mm}$-thick $)$ of dielectric substrate (RT/duroid 6002, $\varepsilon r=2.94, \tan \delta=0.0012)$ separated by an $8 \mathrm{~mm}$-thick dielectric spacer (foam Rohacell $51 \mathrm{HF}$, $\varepsilon \mathrm{r}=1.06, \tan \delta=0.0015)$. A $0.1 \mathrm{~mm}$-thick bonding film is used to glue the RT/duroid 6002 substrates on the foam spacer. To achieve four different LHCP reflected phases (namely a 2-bit phase resolution), four different sets of transverse segments are used. Only one of them is enabled at a time by connecting the three segments forming the resonant wire. In Fig. 3, the active element is represented in red, while the three passives ones are shown in blue. As can be seen in this figure, small gaps are accommodated along the transverse segments in addition to the gaps separating the different transverse segments from the common longitudinal one. These additional gaps are required in order to decrease the parasitic effect of the non-connected transverse segments. In an actual reconfigurable cell, switches would be used to connect the different metallic parts forming a resonant wire. The four possible resonant wires mainly differ from each other in their angular rotation. Indeed, it is well known that the reflected phase of a CP wave can be changed by varying the angular rotation of the reflecting element [5]. As shown in Fig. 3, the final design does not only rely on a rotation of the main resonant wire; the size and shape of the different segments have also been optimised in order to comply with both the technological constraints (available surface allocated to the cell, square grid, etc.) and the foreseen objective (four possible phase states with uniform-distribution in the $\left[0,360^{\circ}\right]$ range at $8.5 \mathrm{GHz}$ ). Figs. 4 to 6 show the three additional cell configurations where resonant wires 2,3 and 4 are activated successively. 


\section{SIMULATIONS}

\section{A. Design}

The frequency responses of these unit-cells have been computed using Ansys-HFSS software (C). In the simulations, we assume that the cell is suspended in an air-filled square metallic waveguide with wave ports at both ends. The need to use a low-permittivity spacer is demonstrated in Fig. 7 which represents the transmission coefficient of a circularlypolarised wave (Fig .7a) and the cross-polarisation reflection coefficient for various values of the permittivity (without any resonant wire). This result makes sense because when the permittivity increases, there is a polarisation conversion induced by parasitic reflection on the dielectric layer. Fig. 7b demonstrates this statement. These numerical results confirm that the unit-cell exhibits better performance with low permittivity spacer. This validates the use of foam as a spacer (Rohacell 51HF). Another important study is the sensitivity in the cell performance when relevant geometrical parameters are varied. The two most critical ones are the following: i) the thickness of the foam spacer, which defines the longitudinal segment's length, ii) the length of the transverse segments on both sides of the spacer. These two parameters must be optimised to achieve a $1 \lambda$-long resonant wire. Figs. 8 and 9 represent the LHCP reflection coefficient for various foam thicknesses and wire lengths. A $300 \mathrm{MHz}$ frequency shift is observed for the maximum deviation $( \pm 2 \mathrm{~mm}$ apart from the optimal values). As a consequence, the proposed unit-cell is considered as rather robust regarding a standard technological PCB fabrication process.

\section{B. Overall performance of the unit-cell}

Figs. 10 and 11 represent the simulated performance for the four possible phase states (optimised design). Fig. 10a shows that the insertion loss for the reflected LHCP is lower than $1 \mathrm{~dB}$ on a [8.2-8.75] GHz range. At the same time, as shown in Fig. 11a, for the four phase-states (in LHCP), the RCHP incident wave is transmitted through the LH-CPSS with insertion loss lower than $1 \mathrm{~dB}$ over a $0.55-\mathrm{GHz}$ range $(6.4 \%)$. The phase of the reflection coefficient in LHCP is plotted in Fig. 10b; the proposed unit-cell exhibits excellent performance. Indeed, four stable phase states are obtained from 8.2 to $8.8 \mathrm{GHz}$, with a nearly uniform distribution in the $\left[0,360^{\circ}\right]$ range. Fig. $11 \mathrm{~b}$ shows that the RCHP wave is transmitted with a nearly constant phase whatever the activated resonant wire. As a conclusion, we show in Fig. 12 the variation of equivalent number of bits (as defined in [6]) versus frequency; these results are very promising since the achieved phase resolution is 1.95 bits.

\section{CONCLUSION}

A new design of CPSS unit-cell has been proposed for dual circularly-polarised reflectarray applications. It provides a nearly 2-bit phase resolution in reflection for the left hand circular polarisation (LHCP) and is almost transparent in right hand circular polarisation (RHCP). Furthermore, the insertion loss for the reflected LHCP and transmitted RHCP is lower than $1 \mathrm{~dB}$ over the [8.2-8.75] GHz range. In order to make this unit-cell reconfigurable, active switches could be used to connect the transverse segments to the longitudinal one to form a continuous resonant wire.

Several unit-cells are under fabrication. The measurement procedure in a waveguide simulator and the experimental data will be presented during the conference. The next task consists in designing the complete reconfigurable unit-cells (as schematized in Fig. 1) so as to reflect independently both orientations of circular polarisation with a controllable phase.

\section{REFERENCES}

[1] M. R. Chaharmir, J. Shaker, M. Cuhaci, and A. Sebak, "Circularly polarized reflectarray with cross-slot of varying arms on ground plane," Electronics Letters, vol. 38, no. 24, pp. 1492-1493, Nov 2002.

[2] B. Strassner, C. Han, and K. Chang, "Circularly polarized reflectarray with microstrip ring rlements having variable rotation angles," IEEE Trans. Antennas Propag, vol. 52, no. 4, pp-1122-1125, Apr. 2004.

[3] E. Girard, R. Moulinet, and R. Gillard, "An FDTD optimization of a circularly polarized reflectarray unit cell," IEEE Antennas Propag. Soc. Int. Symposium, San Antonio, TX, vol. 3, pp. 136-139, Jun. 2002.

[4] J. E. Roy and L. Shafai, "Reciprocal circular-polarization selective surface," IEEE Antennas Propag. Mag., vol. 38, no. 6, pp. 18-33, Dec. 1996.

[5] J. Huang and J. A. Encinar, "Reflectarray antennas," Wiley-Interscience, 2007.

[6] R. Pereira, R. Gillard, R. Sauleau, P. Potier, T. Dousset and X. Delestre, "Robust 2-bit dual-linearly-polarised unit-cell for reflectarray applications," Antennas and Propagation (EUCAP), Proceedings of the $5^{\text {th }}$ European Conference , pp 1488-1490, 11-15 Apr. 2011.

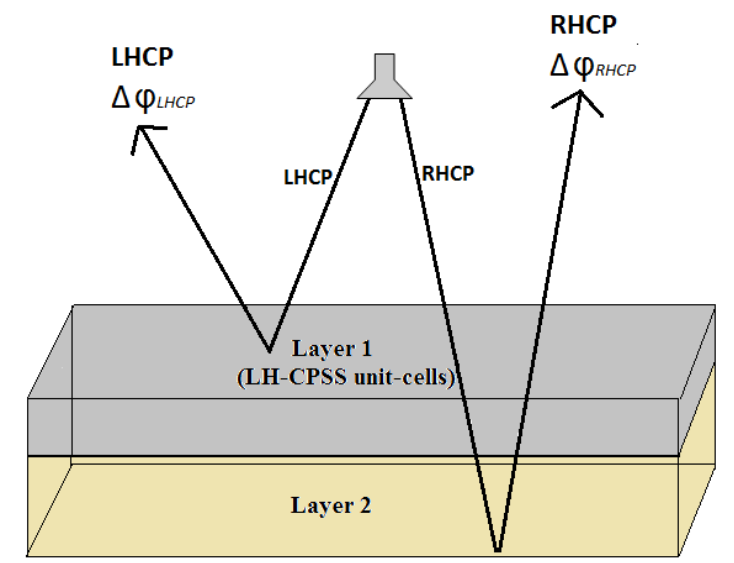

Figure 1. Schematic representation of a reflectarray with independent control of both incident circular polarisations. Only the first layer is studied here; it consists of LH-CPSS unit-cells (Figure 2). 


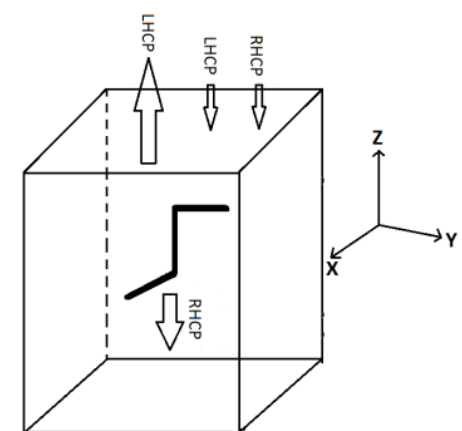

Figure 2. Basic LH-CPSS cell illuminated by a dual circularly-polarised incident wave.

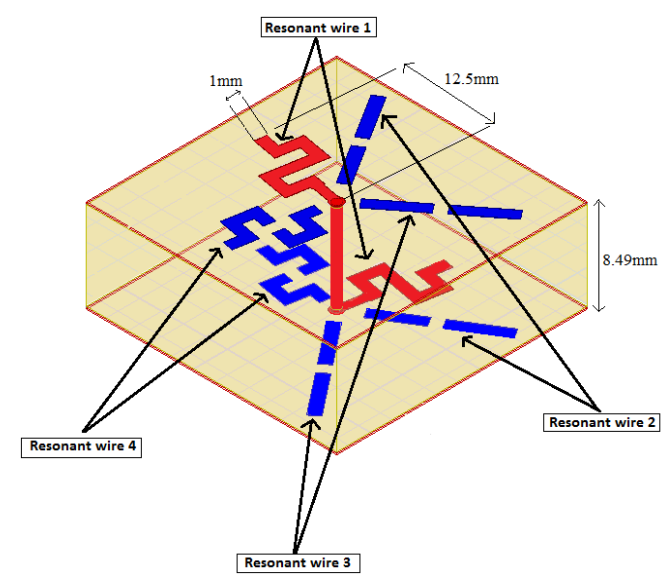

Figure 3. Optimised proposed cell with resonant wire ' 1 ' activated (reflection phase at resonance $=35^{\circ}$ ).

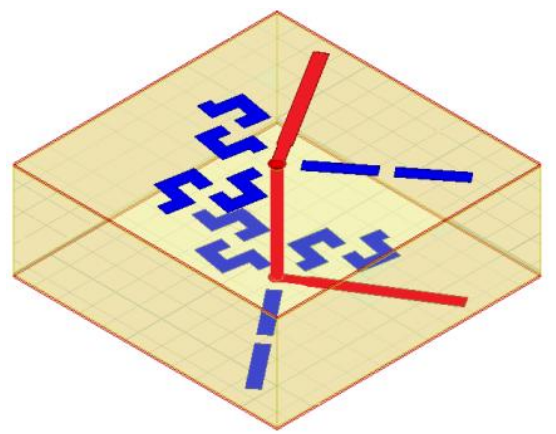

Figure 4. Optimised proposed cell with resonant wire '2' activated (reflection phase at resonance $=125^{\circ}$ ).

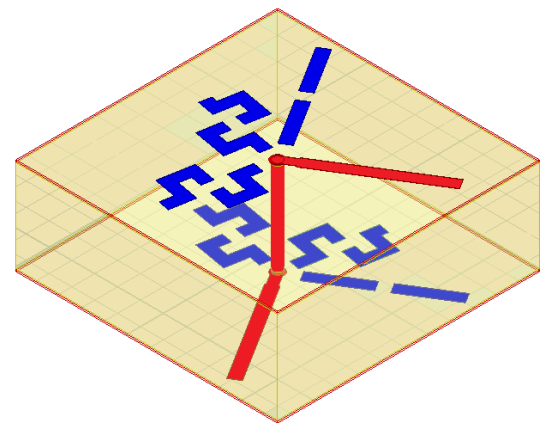

Figure 5. Optimised proposed cell with resonant wire ' 3 ' activated (reflection phase at resonance $=-50^{\circ}$ )

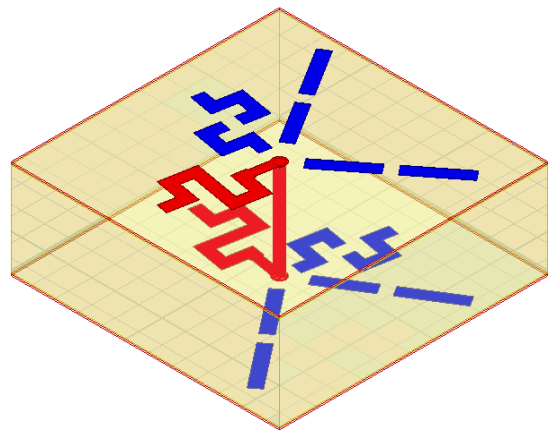

Figure 6. Optimised proposed cell with resonant wire '4' activated (reflection phase at resonance $=-150^{\circ}$ )

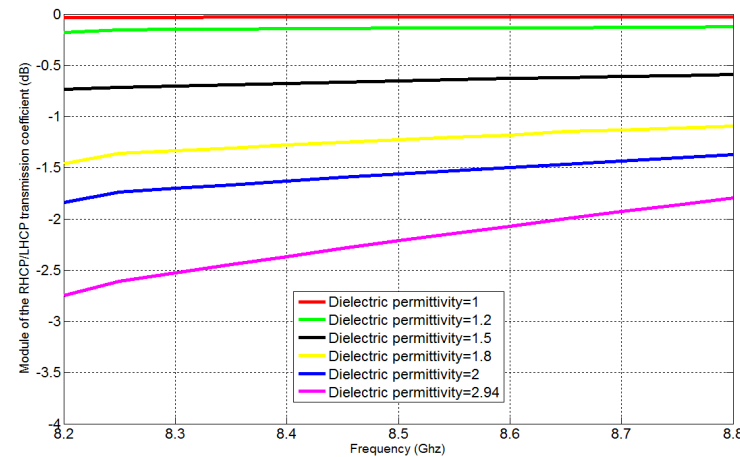

(a)

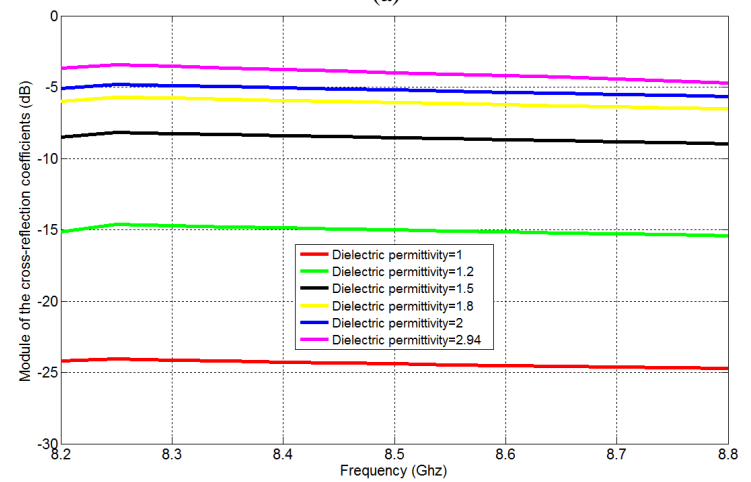

(b)

Figure 7. Amplitude of the (a) RHCP/LHCP transmission coefficient, (b) Cross-reflection coefficient for various permittivity values. In all cases, the dielectric spacer is $8 \mathrm{~mm}$-thick.

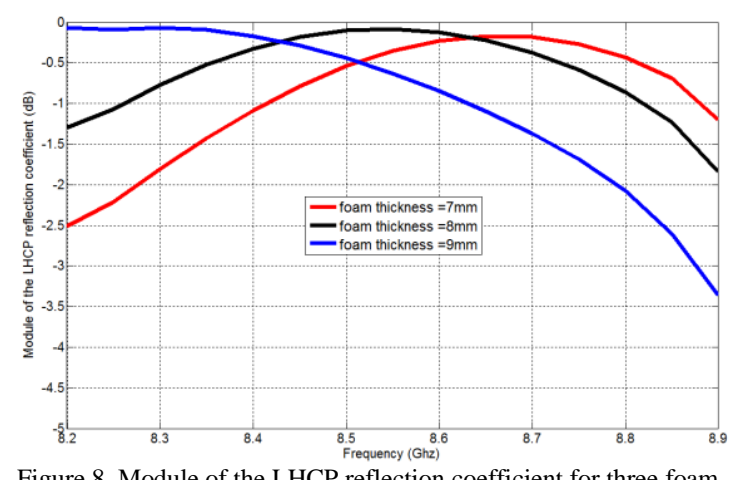

Figure 8. Module of the LHCP reflection coefficient for three foam thicknesses. 


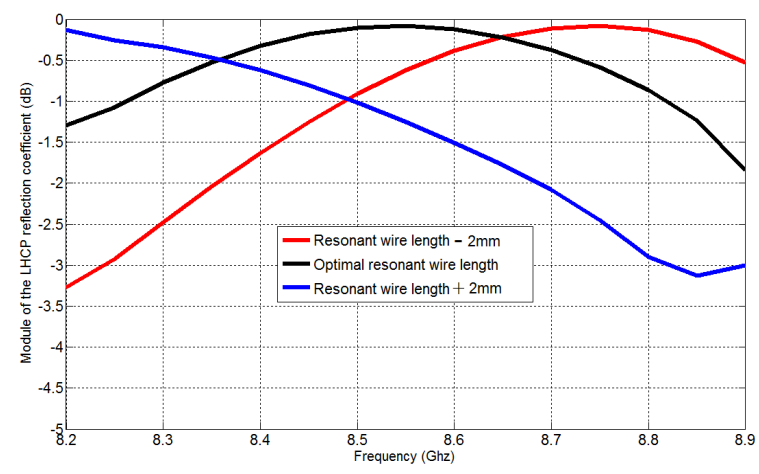

Figure 9. Module of the LHCP reflection coefficient for three resonant wire lengths.

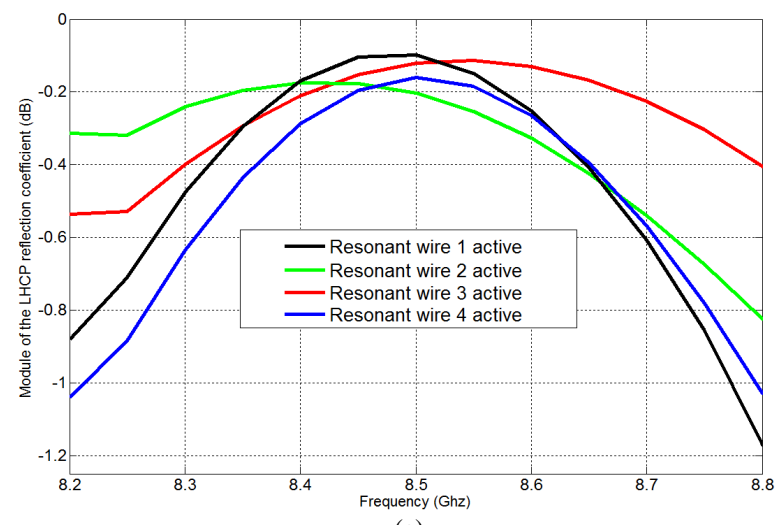

(a)

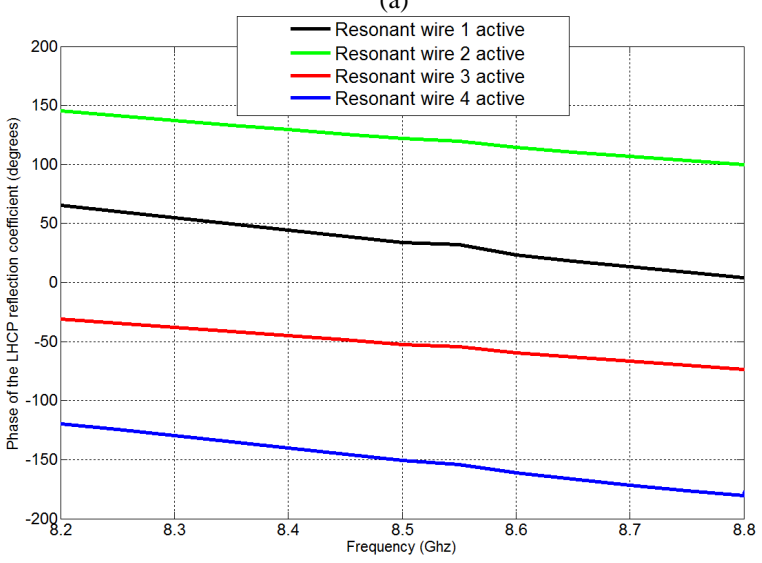

(b)

Figure 10. Reflection coefficient for an incident LHCP wave for the four activated resonant wires of the cell. (a) Magnitude. (b) Phase.

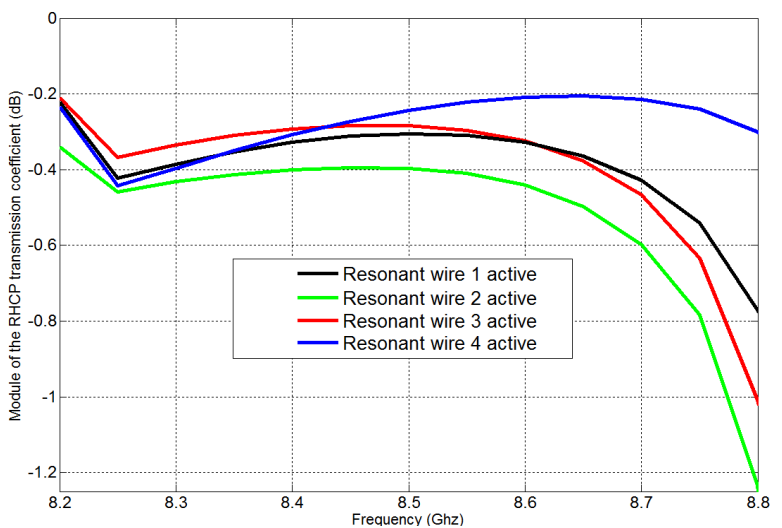

(a)

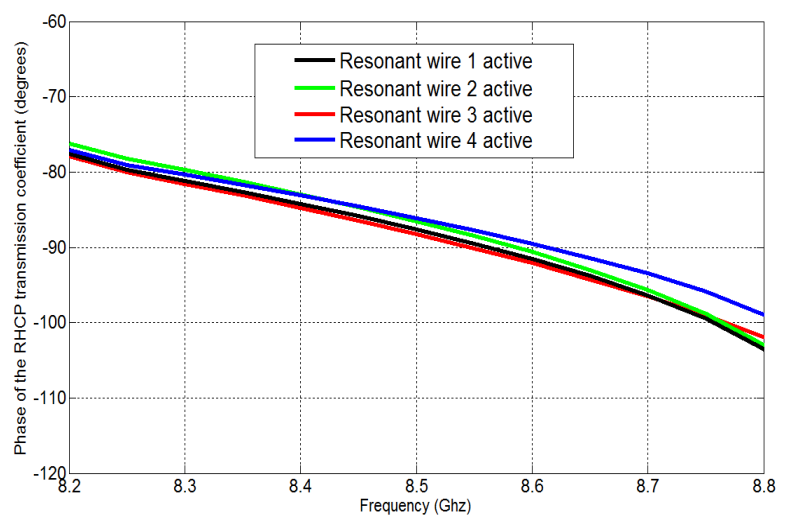

(b)

Figure 11. Transmission coefficient for an incident RHCP wave for the four activated resonant wires of the cell. (a) Magnitude. (b) Phase.

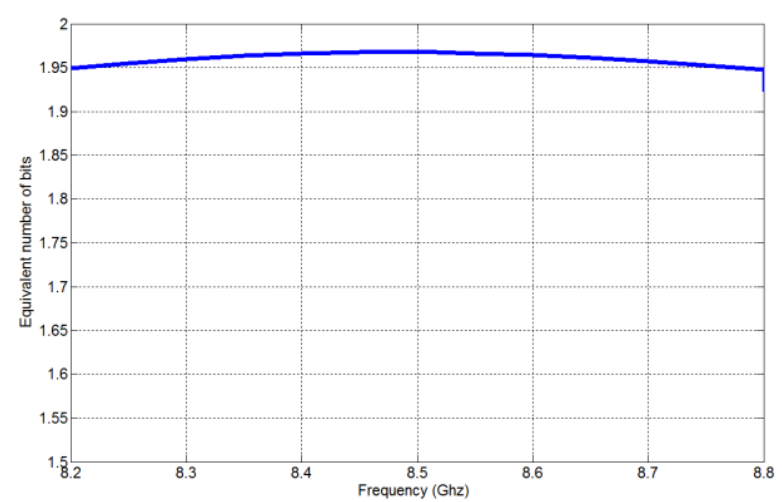

Figure 12. Equivalent number of bits for an incident LHCP 